\title{
Performance Evolution of SC-FDMA for Mobile Communication System
}

\author{
${ }^{1}$ S. B. Lande, ${ }^{2}$ Jyoti D. Gawali and ${ }^{3}$ S. M. Kharad \\ ${ }^{1,2,3}$ E \& EC Department, KITS, Ramtek-441106 \\ landeeed@yahoo.co.in,jyotigawali09@gmail.com,kharadsudhir@gmail.com
}

\begin{abstract}
The main aim of this work is to investigate the performance of Single Carrier Frequency Division Multiple Access (SC-FDMA) in mobile communication system. Nowadays there is increase in demand for high speed data transfer in mobile communication. Different multiple access schemes like Orthogonal Frequency Division Multiplexing (OFDM) and SC-FDM are the two important schemes of Long Term Evolution (LTE) for next generation communication. In this paper, we observe the evolution of SC-FDMA in LTE by showing bit error rate variation and PAPR ratio comparison between OFDMA system and SC-OFDMA system.
\end{abstract}

Keyword: SC-FDMA, OFDMA, 3GPP-LTE, PAPR, BPSK, MATLAB

\section{Introduction}

In next generation wireless communication there is a demand for high data rate transfer with long battery life of user equipment. The SC-FDM is a simplified version of OFDM with low Peak-to-Average Power Ratio (PAPR). SC-FDMA is the uplink transmission scheme in the 3GPP-LTE standard. This interest is justified by the inherent single carrier structure of SC-FDMA, which results in reduced sensitivity to phase noise and a lower PAPR compared to OFDMA [1]. It has become broadly accepted mainly because of its high resistance to frequency selective fading channels [2].

The $3^{\text {rd }}$ Generation Partnership Project (3GPP) is currently specifying the minimum requirements for Long Term Evolution-Advanced (LTE-A) systems. To cope with this ambitious requirement, advanced multiple-antenna techniques, providing high spectral efficiency values and very large spectrum allocations, in the order of $100 \mathrm{MHz}$, are expected to be deployed.

In the previous LTE Release-8 standard [3], OFDMA has been selected for the downlink due to its ability to easily cope with the frequency selectivity of wide-band channels and its high spectral efficiency. In this scheme, the modulated symbols are split over low-rate narrow-band orthogonal subcarriers and a proper Cyclic Prefix (CP) is inserted allowing simple equalization at the receiver [4]. In this paper performance of SCFDMA is shown along with the pilot mapping instead of subcarrier mapping. The performances of our proposed SC-FDMA system are evaluated in both local and wide area scenarios.

\section{Long Term Evolution (LTE)}

Next generation wireless system proposed by $3 \mathrm{GPP}$ is known as LTE. LTE implemented using OFDMA system in downlink and SC-FDMA in uplink communication. This system support both Time and Frequency division duplexing (TDD \& FDD). The example as shown in Figure 1, the generic radio frame has time duration of $10 \mathrm{~ms}$, consisting of 20 slots of each $0.5 \mathrm{~ms}$ [9]. 


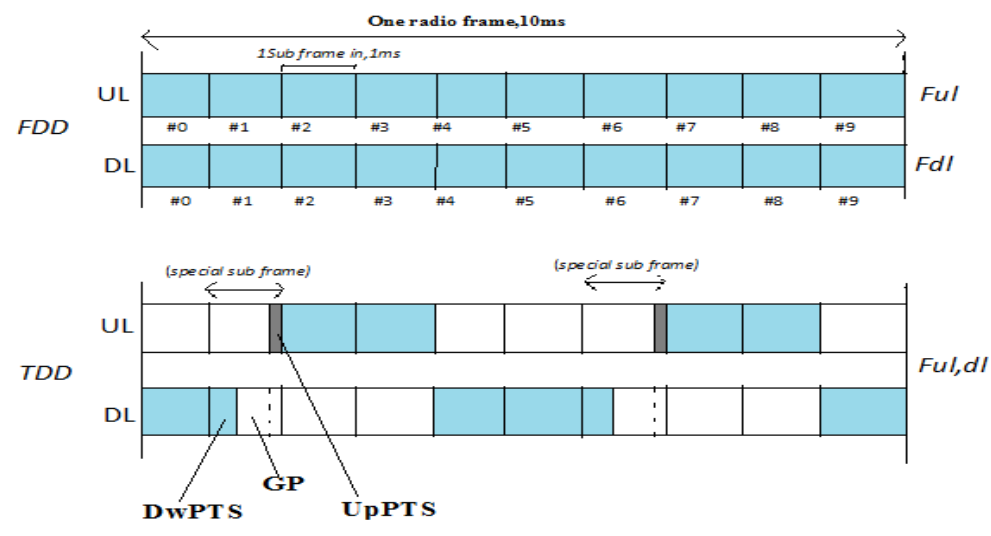

Figure 1. LTE Frame Structure in FDD and TDD Modes [9]

\section{Problem Identification}

Most of the previous communication system based on TDMA, CDMA, FDMA are provides less speed with complex network. The OFDMA system provides high speed but power consumption in devices is more for transmitting data from device in uplink. So we designed SC-FDMA system to whose advantages are listed bellow

- Next generation mobile communication needs high rate of data transfer.

- SC-FDMA allows for the possibility for low-complexity but high-quality equalization in the frequency domain.

- Flexible bandwidth assignments with SC-FDMA.

- Maximizing the utilization of current and future cellular networks. Low sensitivity to carrier frequency offset.

- Less sensitive to non linear distortion and hence, it allows the use of low-cost power amplifiers, low-PAPR (single-carrier property). Greater robustness against spectra nulls.

\section{OFDMA vs. FDMA}

SC-FDMA system contains the advantage of better PAPR. This is, because the DFT block is added at the transmitter to transmit data.

\section{a. OFDM vs Most Used for Broadband Systems}

TDMA \& CDMA technologies are implemented in $2^{\text {nd }}$ and $3^{\text {rd }}$ generation mobile system. Time dispersion depends on multipath fading effect due to transmission of signal through wireless network. In a TDMA and CDMA system, it is the ratio of the channel dispersion to signal symbol time and number of channel taps with strong energy at chiptime resolution over the channel dispersion period respectively. In OFDMA system, channel time dispersion is viewed as the frequency selective or non-selective in the frequency domain called flat fading. In the time domain, the channel is not dispersive relative to its symbol time, and hence, there is no ISI in OFDM system.

\section{b. Sc-Fdma Modulation System:-}

Single carrier modulation, DFT spread orthogonal frequency multiplexing, and frequency domain equalization used in SC-FDMA as OFDMA. In transmitter section block of data are transmitted through modulation block, frequency domain which is the basic property of SC-FDMA using FFT at input, then power \& amplitude levels are assigned using pilot insertion in FFT output signal, IFFT applied, and CP are added for 
error correction, to transmit data through channel $\mathrm{P} / \mathrm{S}$ converter is used at last, serially data transmitted through AWGN channel with Rayleigh fading and inverse operation performed at receiver and get the data which was transmitted at input.

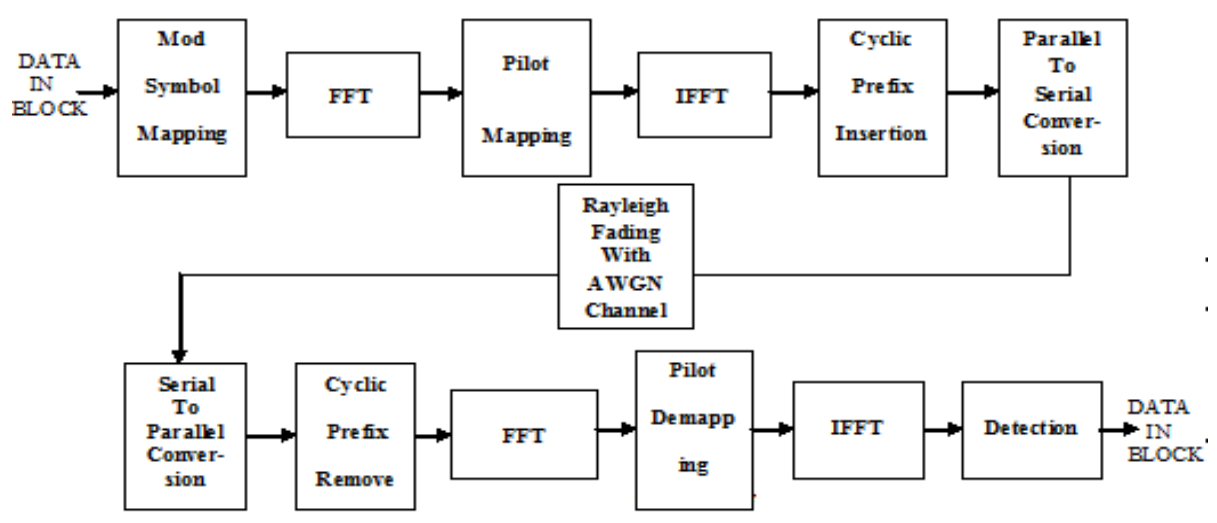

Figure 2. SC-FDMA Transmitter and Receiver

\section{c. OFDMA System}

As SC-FDMA is built over OFDMA modulation, we will look at OFDMA system structure. Typical OFDMA transmitter and receiver structure is shown in Figure 3. An OFDMA symbol is the time used to transmit all of subcarriers that are modulated by block of input data symbols. The modulator transforms the input binary bits into a set of multi-level complex numbers that corresponds to different adaptive modulations formats such as BPSK, QPSK, 16-or 64-QAM. These modulated symbols are then mapped to subcarriers (pilot mapping). An IFFT is used to transform the modulated frequency domain subcarriers into time domain samples.

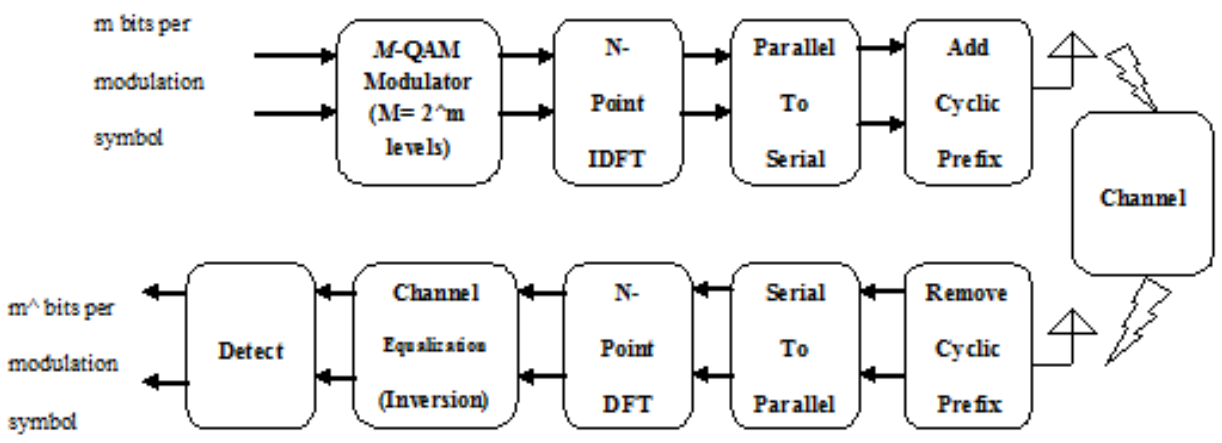

Figure 3. Ofdma System

Having an appropriate modulation format on subcarriers would help to maximize the overall system throughput. A cyclic prefix block copies a portion of the symbol at the end of the time domain samples block to the beginning. Since FFT outputs are periodic hence, copying the samples to the beginning will make the signal continuous. The $\mathrm{CP}$ length depends on the channel delay spread, and is longer than the length of the channel response. At the receiver, the prefix part of the symbol is removed because it may contain ISI from its previous symbol. 


\section{Parameters in OFDMA Based Wireless System}

The SC-FDMA system which we are designed here is based on these parameters of OFDMA system [12].

\section{a. Pilot Placement Scheme:-}

For a time-varying system, pilot symbols become especially important to achieve reliable transmission. Since high information transmission rate makes low percentage of training. Let $\boldsymbol{h}^{\wedge}$ be the channel estimate and MSE is defined as $E\left\{\left\|\boldsymbol{h}^{\wedge}-\boldsymbol{h}\right\|^{2}\right\}$. In [15], $(L+1)$ pilot symbols is optimal. Therefore, in considering the pilot placement schemes

1) The percentage of pilot symbols in the data stream, denoted as $\eta$, is fixed.

2) Each OFDM block size $N=k(l+1), k \geq 1$.

3) $(L+1)$ out of $N$ subchannels are selected periodically as training subchannels from the set $\left\{i, i+\frac{N}{L+1}, \ldots \ldots, i+\frac{L N}{L+1}\right\}, i=0,1, \ldots \ldots, \frac{N}{L+1}-1$. During a training period, pilot symbols in each OFDM block, denoted as $S_{p, m}=\left[S_{p, m}[0], \ldots \ldots, S_{p, m}[L]\right]^{t}$, are sent over the selected $(L+1)$ training subchannel.

4) Pilot symbols have equal power, $\left|S_{p, m}[i]\right|^{2}=\sigma_{p}^{2}$, for any $m$ and $i$.

Since the channel state changes from block to block, we consider the type of periodical placement with equal training cluster size, as shown in Figure 4. For fixed pilot percentage $\eta$, it is not hard to see that given the pilot cluster $r$, the transmission period length $T$ is the given $T=\frac{(L+1) r}{N \eta}$.

Time

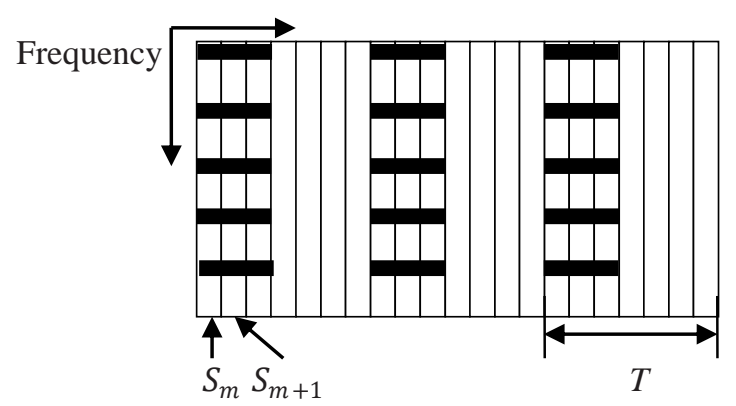

Figure 4. Input OFDM Blocks With Training Period $T$ [15]

\section{b.The Cyclic Prefix Length}

The increase in CP length reduces ISI affect at the expense of higher transmitted power loss. In LTE standard, normal cyclic prefix (around $5.7 \mu \mathrm{s}$ ) and extended cyclic prefix (around $16.67 \mu \mathrm{s}$ ) which corresponds to seven and six OFDM symbols per slot respectively [12].

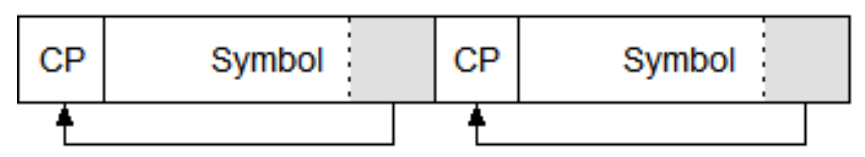

Figure 5. Cyclic Prefix 


\section{c.Performance Calculations}

The performance analysis of OFDMA and SC-FDMA in LTE are characterized by following parameters $[10,14]$.

\section{a. Bit Error Rate (Ber)}

The BER is ratio of errors bits to the total number of bits transmitted during the time interval

$$
\mathrm{BER}=\frac{(\text { error bits })}{(\text { number of transmitted bits })}
$$

\section{b. Signal to Noise Ratio (Snr)}

The SNR is the ratio of bit energy (Eb) to the noise power spectral density (NO) and is expressed in $\mathrm{db}$.

$$
S N R=\frac{\mathrm{Eb}}{\mathrm{N} 0}
$$

\section{c. Ber Vs Snr Process}

For any modulation scheme, the BER is expressed in terms of SNR. BER is measured by comparing the transmitted signal with received signal, and compute the error counts over the total number of bits transmitted.

\section{d. Probability Of Error (Pe)}

The probability of error or error probability $(\mathrm{Pe})$ is the rate of errors occurs in the received signal. For the coherent detection the symbol error probability of M-ary PSK and M-ary QAM in the AWGN channel is determined by following expressions,

For M-ary PSK the Pe is given by

$$
\mathrm{Pe} \cong 2 \mathrm{Q}\left[\sqrt{\frac{2 \mathrm{~Eb} \log _{2} \mathrm{M}}{\mathrm{No}}} \sin \left(\frac{\pi}{M}\right)\right]
$$

Where,

Eblog $2 \mathrm{M}=\mathrm{E}$ (Transmitted signal energy per symbol)

$\mathrm{N} 0=$ Noise density in AWGN

$\mathrm{Q}=\mathrm{Q}$-Function

Therefore

$$
\mathrm{Pe} \cong 2 \mathrm{Q}\left[\sqrt{\frac{2 \mathrm{E}}{\mathrm{No}_{\mathrm{O}}}} \sin \left(\frac{\pi}{\mathrm{M}}\right)\right]
$$

The complementary error function (erfc) used instead of Q. Therefore, the symbol error probability in terms of erfc is

$$
\mathrm{Pe} \cong \operatorname{erfc}\left[\sqrt{\frac{\mathrm{E}}{\mathrm{No}}} \sin \left(\frac{\pi}{\mathrm{M}}\right)\right]
$$

Whereas, the relationship between erfc and Q is given by 


$$
Q(x)=\frac{1}{2} \operatorname{erfc}\left[\frac{x}{\sqrt{2}}\right]
$$

For M-ary QAM the Pe is given by,

$$
P e \cong 4\left(1-\frac{1}{\sqrt{M}}\right) Q\left[\sqrt{\frac{3 E a v}{(M-1) N o}}\right]
$$

Similarly in terms of erfc, the Pe of M-ary QAM is given by

$$
P e \cong 2\left(1-\frac{1}{\sqrt{\mathrm{M}}}\right) \operatorname{erfc}\left(\sqrt{\frac{3 E a v}{2(M-1) N o}}\right)
$$

Where,

N0 $=$ Noise density in AWGN

Eav $=$ Average value of transmitted symbol energy in M-ary QAM or M-ary PSK

\section{e. Peak To Average Power Ratio (Papr)}

The Complementary Cumulative Distribution Function (CCDF) of PAPR is the probability of the PAPR is higher than a certain PAPR value PAPR0 (Pr $\{$ PAPR $>$ PAPR0 $\}$ ).

\section{f. Power Spectral Density}

The power spectral density (PSD) is an important function that describes the power distribution of a signal with respect to frequency.

$$
P_{x x}(m)=\frac{1}{f_{S}}\left\{\frac{1}{N}\left|D F T_{N}\{x(N)\}\right|^{2}\right\}
$$

Where,

$f s=$ Sampling Frequency $(10 \mathrm{MHz})$

$N=$ Number of FFT points $(256=$ total subcarriers $)$

We calculate the PSD at the output of IFFT block in the transmitter of both SC-FDMA.

\section{g. Results and Stimulation}

The stimulation of performance evolution of SC-OFDMA system is implemented in MATLAB.

Table 1. Parameters

\begin{tabular}{|l|l|}
\hline Parameters & Value \\
\hline Number of Subcarrier & 256 \\
\hline CP length & $64(4$ symbol $)$ \\
\hline Modulation Scheme & BPSK, QPSK, 16-QAM, \\
& 64-QAM \\
\hline Data Block size & 16 symbol \\
\hline Bandwidth & $5 \mathrm{MHz}$ \\
\hline
\end{tabular}




\begin{tabular}{|l|l|}
\hline Channel & AWGN (SNR 100db) \\
\hline Fading & Rayleigh (frequency \\
& Selective) \\
\hline & Input sample period = \\
& $1.00 \mathrm{e}-3 \mathrm{sec}$ \\
& Maximum Doppler shift = \\
Rayleigh $\quad$ fading & $100 \mathrm{~Hz}$ \\
parameter & Vector path delays = [0 \\
& 2.00e-5] sec \\
& Average path gain vector \\
& $=[0-9] \mathrm{db}$ \\
\hline FFT and IFFT Size & 64 \\
\hline
\end{tabular}

\section{h. Stimulation Results are Given Below}

Stimulation results are based on different modulation techniques and parameters given in the above table. The results are shown below

\section{g. SC-FDMA and OFDMA Paper Comparison for Different Modulation Scheme}

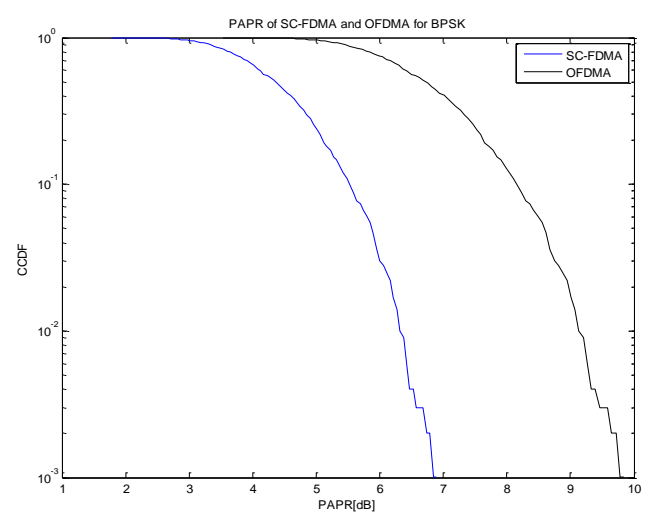

Figure 6. PAPR Comparison for BPSK Scheme

From above Figure 6 we can say that the BPSK modulation scheme have an impact on the PAPR of both OFDMA and SC-FDMA. The overall value of PAPR in SC-FDMA (is shown in figure as blue line) is less than that of OFDMA (is shown in figure as black line).

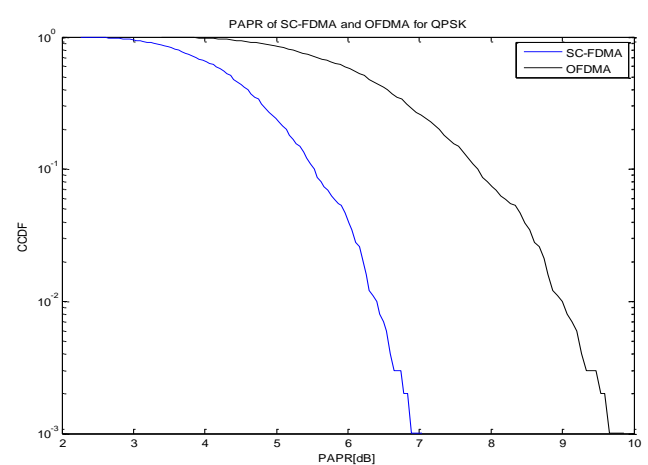

Figure 7. PAPR Comparison for QPSK Scheme 
From above Figure 7 we can Iconclude that the QPSK modulation schemes have PAPR (6.8db) of SC-FDMA and (9.6db) OFDMA system. As the modulation bits increases here than QPSK system that's why it consist of higher PAPR.

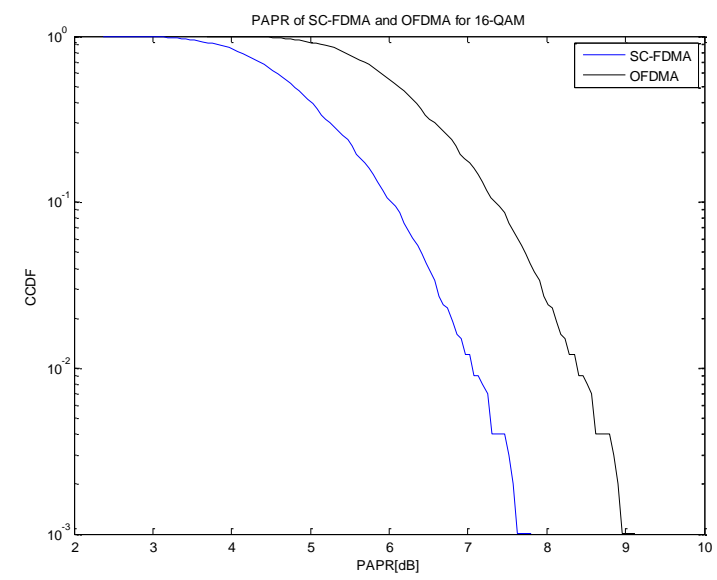

Figure 8. PAPR Comparison for 16-QAM Scheme

Figure 8 shows that there is sharp increase in PAPR of 16-QAM system as the bits transmitted in a system are more than BPSK and QPSK system and have PAPR 7.6db for SC-FDMA system.

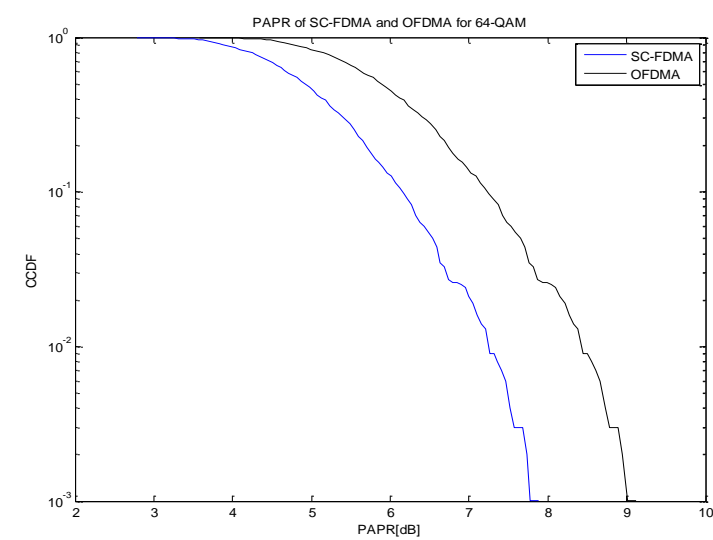

Figure 9. PAPR Comparison for 64-QAM Scheme

From above Figure 9 we can say that the 64-QAM modulation scheme have PAPR than other modulation schemes is $9 \mathrm{db}$ but nearly equal to 16- QAM system.

Table 2. PAPR Comparison

\begin{tabular}{|c|c|c|c|c|}
\hline \multirow{2}{*}{$\begin{array}{c}\text { Modulati } \\
\text { on scheme }\end{array}$} & \multicolumn{2}{|c|}{ SC-FDMA } & \multicolumn{2}{c|}{ OFDMA } \\
\cline { 2 - 5 } & $\begin{array}{c}\boldsymbol{P A P R}(\boldsymbol{B}) \\
\text { BPSK }\end{array}$ & $\begin{array}{c}\boldsymbol{C C} \\
\boldsymbol{D} \boldsymbol{F}\end{array}$ & $\begin{array}{c}\boldsymbol{P A P R}(\boldsymbol{d}) \\
\text { ( })\end{array}$ & $\begin{array}{c}\boldsymbol{C} \boldsymbol{C} \\
\boldsymbol{D} \boldsymbol{F}\end{array}$ \\
\hline $\boldsymbol{Q P S K}$ & 6.85 & $1 \mathrm{e}-3$ & 9.7 & $1 \mathrm{e}-3$ \\
\hline I6QAM & 7.6 & $1 \mathrm{e}-3$ & 9.6 & $1 \mathrm{e}-3$ \\
\hline 64QAM & 7.7 & $1 \mathrm{e}-3$ & 9 & $1 \mathrm{e}-3$ \\
\hline
\end{tabular}


From Table 2, for OFDMA system the PAPR is decreasing continuously if we do for increasing the order of PSK or QAM. But overall PAPR of SC-FDMA is less than that of the OFDMA system. PAPR is the factor which shows power consumption in mobile system. Hence, SC-FDMA is more efficient for uplink communication system than OFDMA

\section{i. Sc-Fdma Ber And Snr For Different Modulation Scheme:-}

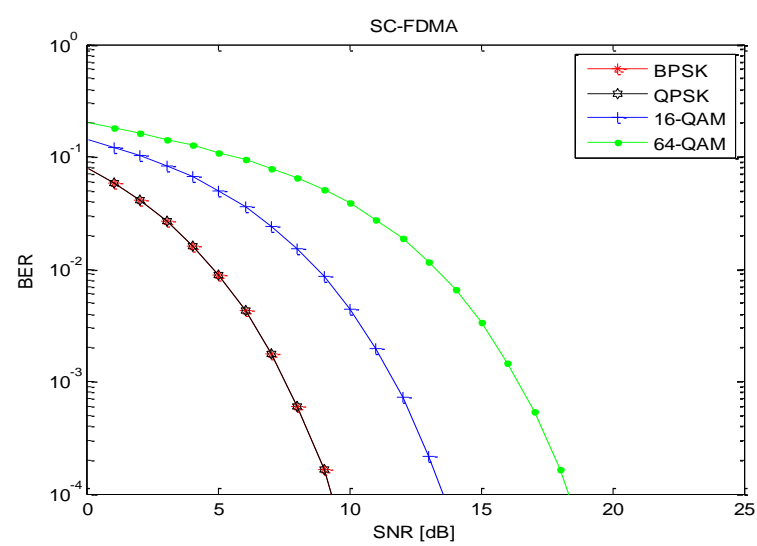

Figure 10. Sc-Fdma Ber Vs Snr

Above Figure10 is the representation of Bit Error Rate along with the Signal to Noise Ratio for SC-OFDMA representing the working of different modulation schemes. Performance of BPSK and QPSK modulation scheme are same as compared to 16-QAM and 64-QAM. When SNR is increasing there is less variation in BPSK and QPSK scheme than that of other modulation scheme.

Table 3. BER vs SNR

\begin{tabular}{|c|c|c|c|}
\hline \multirow{2}{*}{$\begin{array}{l}\text { Modulation } \\
\text { scheme }\end{array}$} & \multirow{2}{*}{$\begin{array}{l}\text { Bits } \\
\text { per } \\
\text { symbol }\end{array}$} & \multicolumn{2}{|c|}{ SC-FDMA } \\
\hline & & SNR & BER \\
\hline BPSK & 1 & 6.8 & $1 e-3$ \\
\hline QPSK & 2 & 6.8 & $1 \mathrm{e}-3$ \\
\hline 16QAM & 4 & 11.7 & $1 \mathrm{e}-3$ \\
\hline 64QAM & 6 & 16.5 & $1 \mathrm{e}-3$ \\
\hline
\end{tabular}

Table III is the representation of BERVs SNR for SC-FDMA representing the working of different modulation schemes. For large value of SNR, there is increasing there is less variation in BPSK and QPSK scheme than that of other modulation scheme.

\section{j. Probability of Error Occurred in Different Modulation Scheme}




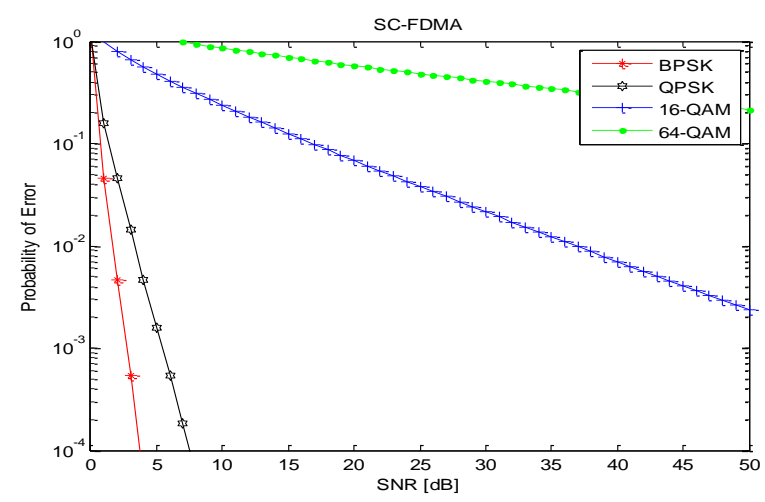

Figure 11. Probability of Error Vs SNR in SC-FDMA System

Figure 11 shows the probability of error occurred in different modulation scheme. The probability of error is high in case of 64-QAM than 16-QAM and QPSK scheme. We can see that BPSK scheme there is very less probability of error when there is change of SNR ratio.

Table 4. Probability of Error

\begin{tabular}{|c|c|c|c|}
\hline \multirow{2}{*}{$\begin{array}{c}\text { Modulatio } \\
\text { n scheme }\end{array}$} & $\begin{array}{c}\text { Bits } \\
\text { per } \\
\text { symbol }\end{array}$ & \multicolumn{2}{|c|}{ SC-FDMA } \\
\cline { 3 - 4 } BPSK & 1 & 1 & $\begin{array}{c}1 \mathrm{P} \text { e- } \\
0.5\end{array}$ \\
\hline QPSK & 2 & 2 & $\begin{array}{c}1 \mathrm{e}- \\
0.5\end{array}$ \\
\hline I6QAM & 4 & 8 & $\begin{array}{c}1 \mathrm{e}- \\
0.5\end{array}$ \\
\hline 64QAM & 6 & 39 & $\begin{array}{c}1 \mathrm{e}- \\
0.5\end{array}$ \\
\hline
\end{tabular}

Probability of error increases with increase in modulation scheme we can see that from Table IV it shows the comparison between all the modulation schemes, where as the bits transmission over symbol increase the probability of error increase.

\section{k. Required Power Spectral Density}

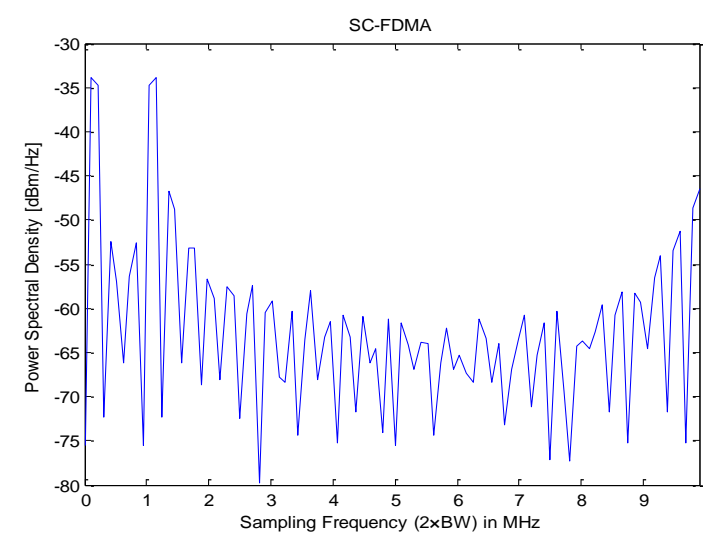

Figure 12. Sampling Frequency Vs Spectral Density 
This Figure 12 shows different sampling frequency required for different power spectral density for of SC-FDMA.

\section{Conclusion}

The PAPR ratio for both the BPSK and QPSK modulation techniques nearly equal for SC-FDMA is varies in the range from $6.5 \mathrm{db}$ to $7 \mathrm{db}$. If we increase the size of QAM the PAPR ratio increases. For SC-FDMA, the PAPR value for 16-QAM \& 64-QAM is in the range $8.5 \mathrm{db}$ to $9 \mathrm{db}$. But the overall value of SC-FDMA is less than OFDMA. Hence SCFDMA must be used for uplink communication system.

The BPSK modulation scheme is having better performance there in performance evolution of SC-FDMA \& QAM modulation schemes are not suitable for uplink communication because there is maximum amount of probability of error increase with increase in SNR. So, they require more power for error correction, hence low order modulation i.e. BPSK, QPSK are adopted in the uplink in order to have less PAPR at user end as observed in result analysis.

\section{References}

[1] R.F. Chisab and C.K. Shukla, "Performance Evaluation of 4G-LTE-SCFDMA Scheme Under SUI and ITU Channel Models", International Journal of Engineering \& Technology IJET-IJENS, (2014) February.

[2] R.F. Chisab and Professor(Dr.) C. K. Shukla, "Comparative Study in Performance for Subcarrier Mapping in Uplink 4G-LTE under Different Channel Cases", International Journal of Advanced Computer Science and Applications, vol. 5, no. 1, (2014).

[3] "LTE Physical Layer-General Description (Release 8)", $3^{\text {rd }}$ Generation Partnership Project, Technology Rep. TS 36201, version 8.1.0, (2007).

[4] L. Hanzo, M. Munster, B. Choi and T. Keller, "OFDM and MCCDMA for Broadband Multi User Communications, WLANs and Broadcasting", John Wiley-IEEE Press, (2003).

[5] R. Agraval, R. Berry, J. Huang and V. Subramanian, "Optimal Scheduling for OFDMA Systems", Fortieth Asilomar Conference on Signals, Systems and Computers (ACSSC'06), (2006), pp. 1347-1351.

[6] C. Wengerter, J. Ohlhorst and A.G.E. von Elbwart, "Fairness and Throughput Analysis for Generalized Proportional Fair Frequency Scheduling in OFDMA", IEEE Proceedings of the 61th Vehicular Technology Conference, vol. 3, (2005), pp. 1903-1907.

[7] A. Pokhariyal, "Frequency Domain Packet Scheduling Under Fractional Load for the UTRAN LTE Downlink", IEEE 65th Vehicular Technology Conference, (2007), pp. 699-703.

[8] J. Lim, G. Myung, K. Oh and D.J. Goodman, "Channel Dependent Scheduling of Uplink Single Carrier FDMA Systems", IEEE Proceedings of the Vehicular Technology Conference, (2006), pp. 1-5.

[9] F.D. Calabrese, "Search-Tree Based Uplink Channel Aware Packet Scheduling for UTRAN LTE", IEEE Proceedings of the 67th Vehicular Technology Conference, (2008).

[10] T. Satio, Y. Tanaka and T. Kato, "Trends in WiMaX/LTE system", Fujitsu scientific and technical journal, vol. 45, (2009), pp. 355-362.

[11] J. Lee, J.K. Han and J.H. Zhang, "MIMO Technologies in 3GPP LTE and LTE Advanced", EURASIP Journal on Wireless Communications and Networking, (2009).

[12] P.H. Lehne and F. Bohagen, "OFDMA for wireless communication", R\&I Research Report, Telnor, (2008).

[13] H.G. Myung and D.J. Goodman, "Single Carrier FDMA: A New Air Interface for Long Term Evolution", John Wiley \& Sons Ltd, (2008).

[14] "Technical White Paper: Long Term Evolution (LTE): A Technical Overview", Motorola, Inc. www.motorola.com, (2007).

[15] M.D. and L.Tang and B.M. Sadler, "Optimal Pilot Placement for Channel Tracking in OFDM", Army Research Office and Multidisciplinary University Research Initiative, (2000). 
International Journal of Future Generation Communication and Networking Vol. 9, No. 2 (2016) 